\title{
Infrared-Triggered Actuators from Graphene-Based Nanocomposites
}

\author{
Jiajie Liang, Yanfei Xu, Yi Huang,* Long Zhang, Yan Wang, Yanfeng Ma, Feifei Li, \\ Tianying Guo, and Yongsheng Chen*
}

Key Laboratory of Functional Polymer Materials and Center for Nanoscale Science \& Technology, Institute of Polymer Chemistry, College of Chemistry, Nankai University, Tianjin 300071, China

Received: February 11, 2009; Revised Manuscript Received: April 18, 2009

\begin{abstract}
The emerging field of optical-triggered actuators based on polymeric nanocomposite continues to be the focus of considerable research in recent years because of their scientific and technological significance. In principle, dispersing nanofiller with unique characteristics in polymer matrix can not only provide superb enhancement of performance but also afford novel actuation schemes to the systems. Graphene, combining its unusual electrical, thermal, mechanical, and optical properties, can provide the ability to act as "energy transfer" and trigger unit in the realm of nanocomposite actuators. Herein, we demonstrate a new dimension to this 2D nanoscale material by showing the excellent light-triggered acutation of its thermoplastic polyurethane nanocomposites with significantly enhanced mechanical properties. These nanocomposite actuators with 1 wt \% loading of sulfonated functionalized graphene sheets (sulfonated-graphene) exhibit repeatable infraredtriggered actuation performance which can strikingly contract and lift a $21.6 \mathrm{~g}$ weight $3.1 \mathrm{~cm}$ with $0.21 \mathrm{~N}$ of force on exposure to infrared light and demonstrate estimated energy densities of over $0.33 \mathrm{~J} / \mathrm{g}$. Some cases can even reach as high as $0.40 \mathrm{~J} / \mathrm{g}$. Dramatic improvement in mechanical properties is also obtained for the graphene nanocomposites with homogeneous dispersion. As the concentration of sulfonated-graphene increases, its nanocomposites show significantly enhanced mechanical properties, that is, the Young's modulus increases by $120 \%$ at only $1 \mathrm{wt} \%$ loading. Moreover, through comparative study of three kinds of graphene materials, it is found that this infrared-triggered actuation property is principally dependent on the integrity of the aromatic network of graphene and on its dispersion state within the matrix.
\end{abstract}

\section{Introduction}

Actuation, that is, the ability of materials to undergo largescale shape or mechanical property changes in response to an appropriate external stimulus, has continued to grab considerable attention of both scientists and engineers. ${ }^{1,2}$ Current actuator materials, which can be classified into electrical, thermal, pneumatic, and optical actuators depending on their corresponding energy supplies, ${ }^{3,4}$ have been widely adopted in a variety of technological uses, including temperature-sensitive switches, ${ }^{2}$ microrobotics, ${ }^{5}$ artificial muscle,,${ }^{1,6-8}$ and even molecular machines. ${ }^{9} 10$ Although many electrical-triggered actuator materials, such as dielectric elastomers and conducting polymers, ${ }^{8,11,12}$ are well-known and are used in a wide range of frontier technologies, they are still handicapped in some practical applications by high voltages, electric wires fixed in the devices, and so on. ${ }^{3}$ Correspondingly, an optical-induced actuator, compared to the actuators driven by other stimuli, offers an alternative means to couple energy remotely into actuator structures, which brings larruping advantages such as wireless actuation, remote control, and high-level integrity. ${ }^{3}$ Specially, significant progress has been made recently in the development for polymer-based optical-triggered actuator materials because of the polymer material's light weight, good processability, and low cost and particularly because it has stroke, force, and efficiency similar to that of human muscles. ${ }^{3}$ These advantages make the optical-triggered polymeric actuators promising for a variety of applications especially in the field of medical devices where triggers other than heat or electricity are highly desirable. ${ }^{9}$

* To whom correspondence should be addressed. E-mail: yschen99@ nankai.edu.cn (Y. C.).
Currently, two types of light-triggered polymeric actuators have been reported including pure polymers ${ }^{9,13,14}$ and polymer composites. ${ }^{1,2,9,15,16}$ In either of these cases, these polymeric materials have to be equipped with photoactive functional groups or fillers. That is, both types of actuator materials need to consist of two components on the intra- or intermolecule level: "molecular switch" and "energy transfer" units, where the former works as the mechanical deformation (molecular switch) unit and the latter acts as the trigger (energy transfer) unit to transfer the external light energy to the molecular switch unit. ${ }^{14}$ Nevertheless, many optical-triggered pure-polymeric actuators excel in some actuation performance (such as energy density) but are unsatisfactory in others (i.e., mechanic performance and response times). ${ }^{9}$ Several advantages of composite-based actuation materials are worthy of attention. These include (1) overall mechanical enhancement of the matrix material if an appropriate filler (as the energy-transfer component) is chosen and (2) better or novel characteristics may be derived from these composites, such as salient thermal stability and electrical conductivity. With this strategy, the addition of distinctly different nanofillers to polymeric actuator matrix may exploit synergism between the nanofillers and the polymers and may impart a novel and prominent actuation behavior as well as superb enhancement of overall performance. ${ }^{1,2,15,16}$

Thermoplastic polyurethane (TPU), which possesses the ability to store and efficiently recover large strains by application of prearranged thermal stimuli because of its two-phase structure: a thermally reversible phase responsible for fixing a transient shape and a frozen phase responsible for recovering the original shape, ${ }^{1,6,7,17,18}$ is one of the most widely used polymeric thermal-induced actuator materials. Unfortunately, 
TPU, which is essentially infrared (IR) transparent, does not show IR-induced actuation. ${ }^{1}$ Recently, making use of their outstanding electrical, thermal, mechanical, and optical properties of carbon nanotubes (CNTs), a CNT/TPU nanocomposite has been used for an actuator showing remote, rapid, and highenergy infrared-induced actuation behavior along with remarkable enhancement of mechanical performance. ${ }^{1}$ With an average dimension of about $20 \times 4 \times 0.4 \mathrm{~mm}$, the CNT/TPU film could contract and lift a $60 \mathrm{~g}$ weight $3.3 \mathrm{~cm}$ on exposure to IR irradiation. However, the inherent bundling of carbon nanotubes, their intrinsic impurities from catalysts and amorphous carbon, their poor dispersibility, and their high cost have been hampering their practical application to date.

Graphene, one atom thick and two-dimensional (2D) single layer, is emerging as a rising star in the field of material science because of its prominent intrinsic properties, which may rival or even surpass both the single and multiwalled carbon nanotubes. ${ }^{19-27}$ In addition to its extraordinary electronic transport properties, ${ }^{19-22}$ both recent theoretical ${ }^{23}$ and experimental ${ }^{24-27}$ results have revealed that graphene is the strongest material developed so far. ${ }^{27}$ Thus, nanocomposites utilizing graphene materials as nanofillers are offering opportunities to impart unprecedented enhancing mechanical and thermal properties to polymers at very low loadings. ${ }^{24}$ Moreover, the perfect $\mathrm{sp}^{2}$ carbon-network structures of the graphene materials ensure them to have eximious thermal conductivity and IR response. ${ }^{28-30}$ Given these combined and unique properties of graphene materials as well as the thermal-active characteristic of TPU, we expect that graphene/TPU nanocomposites, where graphene can work as photoactive energy transfer and TPU can act as molecular switch units, would be imparted with salient IRtriggered acutation behavior together with significantly enhanced mechanical performance.

The latest results ${ }^{16,21,24}$ including our own studies ${ }^{31}$ have demonstrated that the efficacious use of graphene in polymer nanocomposites is strongly correlated with the ability to disperse them homogeneously in a polymer matrix without destroying their integrity of graphitic network. Perfect graphene does not exist naturally, but bulk and aqueous or organic solution processable functionalized graphene materials can now be prepared easily. ${ }^{23,24,32-36}$ These chemical functionalizations have been found to be a feasible and effective means for improving the dispersion of graphene in polymer matrix. ${ }^{21,24}$ However, the damage to graphene's $\mathrm{sp}^{2}$ carbon network and the functionality groups attached on the graphene sheets which act as "defect" sites would severely affect the properties of graphene, such as mechanical ${ }^{25}$ electrical, ${ }^{21}$ thermal, ${ }^{30}$ and optical absorption properties. $^{28}$ Subsequently, the performance of the nanocomposites using the heavily functionalized graphene directly is limited. The removal of such defect groups/sites could (partially) restore the aromatic network of graphene, but it could also result in agglomeration and precipitation of the graphene sheets if no force, such as the presence of polymer media, prevents it. ${ }^{21,34}$ Therefore, graphene materials that can be well dispersed in solvent (and polymer matrix) but with light chemical modification and essentially retained extensive conjugated $\mathrm{sp}^{2}$ carbon network are needed for our purpose. Recently, it was reported that sparingly sulfonated functionalized graphene (sulfonatedgraphene), prepared with prereduction of graphene oxide (GO) followed by a diazonium reaction for attachment of $\mathrm{SO}_{3} \mathrm{H}$ group and then a postreduction with hydrazine, exhibits good water solubility and structurally, largely restored conjugated $\mathrm{sp}^{2}$ carbon network with excellent conductivity. However, this material only has limited solubility in organic solvents. ${ }^{37}$ Thus, following this direction, we have modified the procedure in this literature (see Supporting Information, SI-3) and have developed a modified sulfonated-graphene material with better solubility in some common organic solvents, such as $\mathrm{N}, \mathrm{N}$-dimethylformamide (DMF), as determined by atomic force microscopy (AFM) (see below). Indeed, this sulfonated-graphene has an extensively conjugated $\mathrm{sp}^{2}$ carbon network, and the intrinsic properties of graphene have been largely restored as determined by its conductivity test ${ }^{37,38}$ and IR absorption as well as thermogravimetric analysis (TGA) results in our measurements below. Hence, in this investigation, sulfonated-graphene was homogeneously incorporated into TPU (Irogran PS455-203) matrix, and a remarkable light-triggered actuation as well as a dramatic enhancement of mechanical property was obtained for its nanocomposites.

For a comparative purpose, two other graphene materials were also investigated: isocyanate treated graphene oxide (isocyanategraphene), ${ }^{36}$ which has good solubility in organic solvents and good dispersion in polymers but a rather damaged $\mathrm{sp}^{2}$ carbon network, and fully reduced graphene-based sheets (reducedgraphene), ${ }^{38}$ which have an excellent restored $\mathrm{sp}^{2}$ carbon network but almost no solubility in most solvents and rather poor dispersion in polymer matrix. All three kinds of graphene materials were incorporated into TPU matrix using similar solution processing methods as detailed in section 2.2.

\section{Experimental Section}

2.1. Raw Materials. Graphite was obtained from Qingdao Huarun graphite Co., Ltd., with a particle size of $20 \mu \mathrm{m}$. Hydrazine hydrate $(80 \%)$ was purchased from Tianjin Ruijinte Chemical Co., Ltd. Sulfanilic acid was supplied by the Shanghai Chemical Co., Ltd.

The thermoplastic polyurethane (TPU, Irogran PS455-203) was purchased from Huntsman Polyurethanes. This linear segmented polyurethane exhibits a low-glass-transition temperature $\left(T_{\mathrm{g}}=-36.5{ }^{\circ} \mathrm{C}\right.$, determined by dynamic mechanical analysis, as the temperature of the maximum in $\tan \delta$ ), nearambient melting of soft-segment crystallites $\left(T_{\mathrm{m}, \mathrm{s}}=41{ }^{\circ} \mathrm{C}\right.$, determined by differential scanning calorimetry (DSC)), and high deformation (ca. 650\%, measured at a crosshead speed of $100 \mathrm{~mm} / \mathrm{min}$ ). ${ }^{18}$ The hard-segment crystallites provide the reversible physical cross-links that render polyurethane a thermoplastic elastomer. Strain-induced crystallization of the soft segments enhances mechanical performance under extreme deformation by increasing physical cross-link density and by providing reinforcement.

2.2. Procedure for Processing of Nanocomposites. Three kinds of nanocomposites were all fabricated by a solution mixing process. In brief, the desired amount of isocyanate-graphene, sulfonated-graphene, or reduced-graphene was dispersed in DMF with the aid of sonication for $1-3 \mathrm{~h}$, and a requisite amount of TPU in DMF solution was gradually added under constant stirring. Then, the mixtures were sonicated for an additional $30 \mathrm{~min}$ and were poured into a Teflon Petri dish to let the solvent evaporate for film formation. Those yielding films were peeled off and were cut into strips of $\sim 30 \mathrm{~mm} \times 5 \mathrm{~mm}$ $\times 0.05 \mathrm{~mm}$ for further testing. The preparation process for the sample with $1 \mathrm{wt} \%$ loading of sulfonated-graphene is detailed in the Supporting Information, SI-5.

2.3. Characterization. Typical tapping-mode atomic force microscopy (AFM) measurements were performed using Multimode SPM from Digital Instruments with a Nanoscope IIIa Controller. Samples for AFM images were prepared by depositing the dispersion of sulfonated-graphene or isocyanate-graphene 

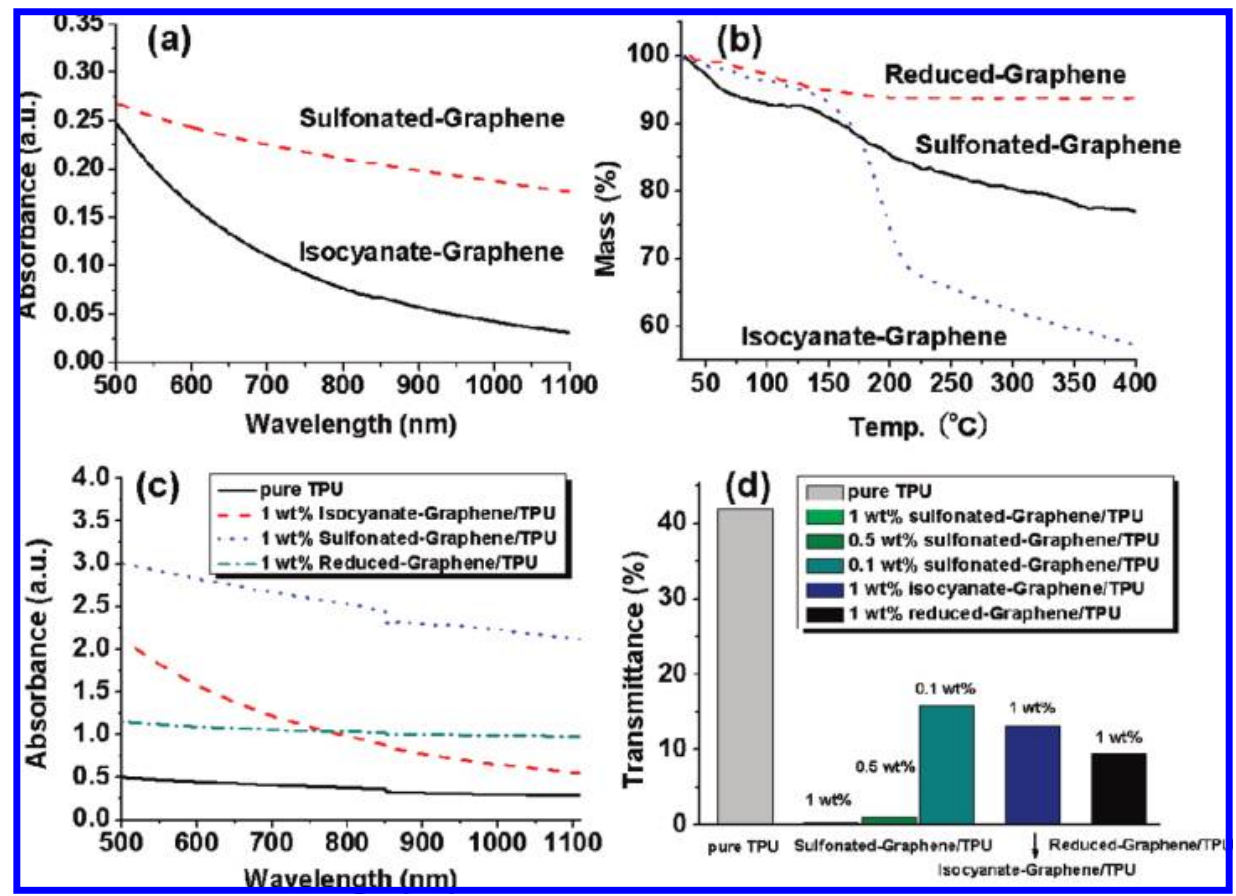

Figure 1. IR absorption property of the three graphene materials and their nanocomposites. (a) IR absorption properties of sulfonated-graphene and isocyanate-graphene solutions with concentration of $0.05 \mathrm{mg} / \mathrm{mL}$. As the reduced-graphene is insoluble in DMF, its IR absorption spectrum is not shown here. (b) The normalized IR absorption of the films of pure TPU, isocyanate-graphene/TPU (1 wt \%), sulfonated-graphene/TPU (1 wt $\%$ ), and reduced-graphene/TPU (1 wt \%) across a range of wavelength from 500 to $1100 \mathrm{~nm}$. (c) Summary of the transmittance of IR light for the sample films at $850 \mathrm{~nm}$ : pure TPU, isocyanate-graphene/TPU (1 wt \%), sulfonated-graphene/TPU $(0.1,0.5$, and $1 \mathrm{wt} \%)$, and reduced-graphene/ TPU $(1 \mathrm{wt} \%)$. All the sample films, prepared from the solutions with the same concentration $(1 \mathrm{~g}$ TPU in $7 \mathrm{~mL}$ DMF as seen in the Supporting Information, SI-5), have the same thickness of $\sim 0.05 \mathrm{~mm}$ for these IR absorption measurements. The light transmittance ( $T$ ) of the sample films can be gained from the formula absorption units $(\mathrm{Au})=\log [1 / T]$ as the thickness of the films are given. (d) The TGA curves with heating rate of $5{ }^{\circ} \mathrm{C} / \mathrm{min}$ from room temperature to $400{ }^{\circ} \mathrm{C}$ under $\mathrm{N}_{2}$ for isocyanate-graphene, sulfonated-graphene, and reduced-graphene.

in DMF $(0.2 \mathrm{mg} / \mathrm{mL})$ (graphene oxide in $\mathrm{H}_{2} \mathrm{O}$ ) onto a freshly cleaved mica surface and allowing them to dry in air. Scanning electron microscopy (SEM) was performed on LEO 1530 VP field emission scanning electron microscope with an acceleration voltage of 5-10 kV. Samples were prepared by immersing the films in liquid nitrogen for $10 \mathrm{~min}$ before fracture. Thermogravimetric analysis (TGA) was carried out using a NETZSCH STA 409PC instrument at a heating rate of $5^{\circ} \mathrm{C} / \mathrm{min}$ from room temperature to $1000{ }^{\circ} \mathrm{C}$ under $\mathrm{N}_{2}$. The NIR/IR spectra were obtained with a JASCO V-570 spectrometer in the 200-2000 $\mathrm{nm}$ range adjusted for the background.

2.4. Infrared Actuation and Mechanical Measurement. For infrared actuation, we used $\mathrm{Hg}-\mathrm{Xe}$ lamp (with a red filter with cutoff $<600 \mathrm{~nm}$ ) as the light source positioned $30 \mathrm{~cm}$ from the samples. The power density delivered to the sample was measured at $\sim 30 \mathrm{~mW} / \mathrm{cm}^{2}$ using the light intensity meter (Beijing Normal University Photoelectric Apparatus Co., Ltd., Model: FZ-A). To avoid the impact of thermomechanical history and crystallite distribution, samples were first elongated to $200 \%$ of the original length and then were heated to relax before IR actuation was carried out. The mechanical properties of these nanocomposites were measured by a universal tensile testing machine (KNM 500-10 test metric, United Kingdom) at $23{ }^{\circ} \mathrm{C}$ with $50 \%$ relative humidity. The extension rate was $20 \mathrm{~mm} /$ min, and the load cell was $250 \mathrm{~N}$ with a gauge length of $\sim 20$ $\mathrm{mm}$. In all cases, more than five samples were tested from which the mean and standard deviation were calculated. Dimensions of the samples for infrared actuation and mechanical measurement were both $\sim 30 \mathrm{~mm} \times 5 \mathrm{~mm} \times 0.05 \mathrm{~mm}$.

\section{Results and Discussion}

Investigations of the IR absorption properties of these three graphene materials were first carried out to test their IR response for actuation purpose as shown in Figure $1 \mathrm{a}-\mathrm{c}$. The IR/NIR absorption for the DMF solutions (Figure 1a) of sulfonatedgraphene and isocyanate-graphene shows that, while both the sulfonated-graphene and isocyanate-graphene solutions have good optical absorption in the 600-1100 nm wavelength range at the same concentration $(0.05 \mathrm{mg} / \mathrm{mL})$, the sulfonatedgraphene shows much stronger absorption in this region. For example, at 600, 800, and $1000 \mathrm{~nm}$, the absorbance of sulfonated-graphene solutions is $150 \%, 270 \%$, and $440 \%$ of that for the isocyanate-graphene solutions, respectively. The solution absorption of reduced-graphene was not obtained because of its poor solubility in DMF. The IR absorption of their TPU nanocomposites was further investigated as presented in Figure 1b. Clearly, sulfonated-graphene/TPU nanocomposite outperforms reduced-graphene/TPU and isocyanate-graphene/TPU with respect to the IR absorption at the same loadings. Furthermore, as depicted in Figure 1c about the transmittance of IR light ${ }^{15}$ at $850 \mathrm{~nm}$ for TPU and the nanocomposite films with the same thickness, pure TPU film shows a transmittance of $43.0 \%$, which is much higher than those of the nanocomposite films. The sulfonated-graphene/TPU with 1 wt $\%$ loading transmits only $0.3 \%$ of light at the wavelength of $850 \mathrm{~nm}$. This is significantly lower than that of reduced-graphene/TPU $(9.5 \%)$ and isocyanate-graphene/TPU nanocomposites (13.2\%). Moreover, only $1.0 \%$ light has been transmitted for the sulfonatedgraphene/TPU with 0.5 wt \% loading of sulfonated-graphene, which is even much lower than that of reduced-graphene/TPU and isocyanate-graphene/TPU nanocomposites with 1 wt $\%$ loading.

It is believed that two main distinct characters, the level of restoration of carbon $\mathrm{sp}^{2}$ network and the ability of homogeneous dispersion for graphene materials in the TPU matrix, lead 


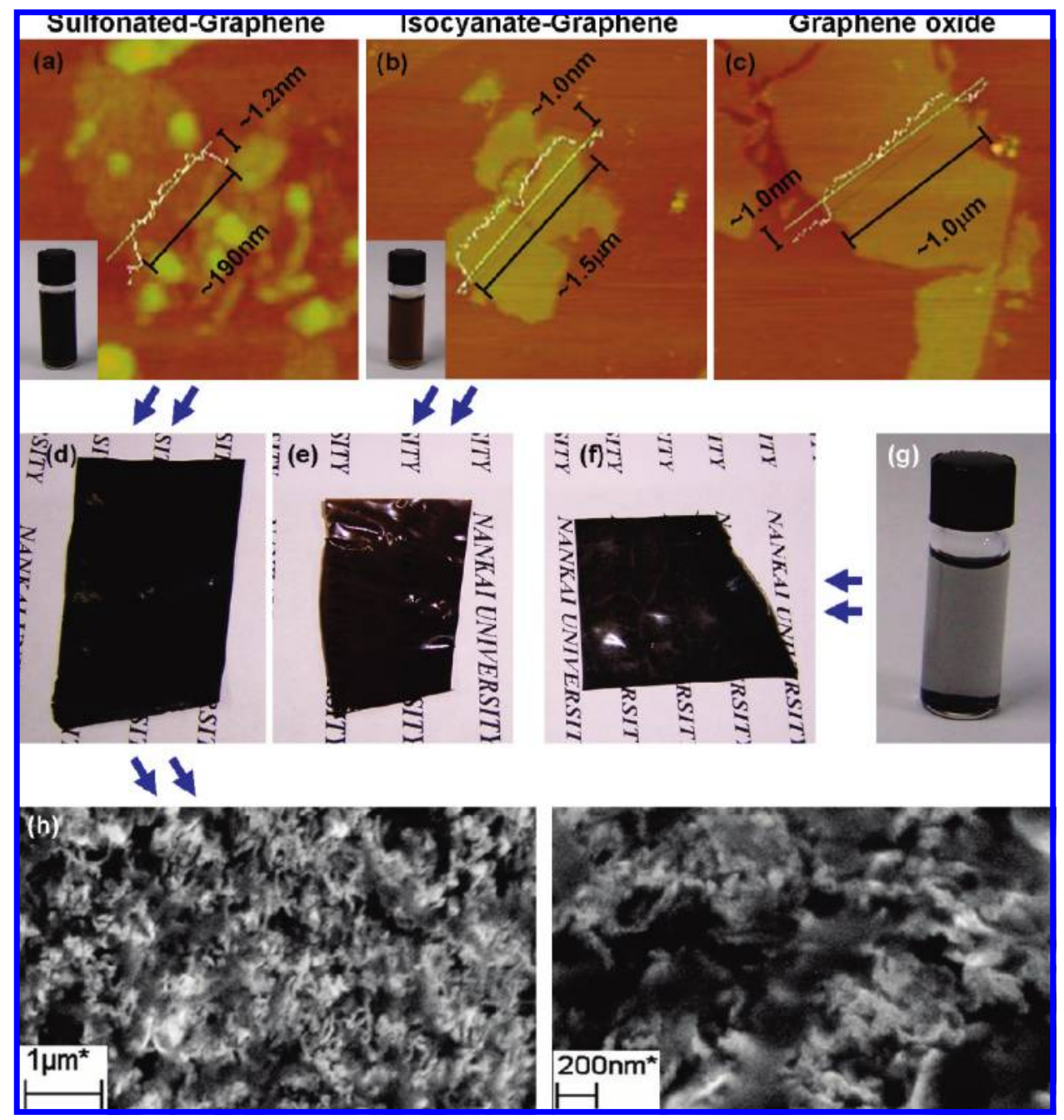

Figure 2. Microscopy and optical images for the graphene materials and their nanocomposites. (a) A typical tapping-mode AFM image of sulfonatedgraphene sheets deposited on mica substrate from DMF dispersion (inset) with superimposed cross section measurements taken along the white line indicating a sheet thickness of $\sim 1.2 \mathrm{~nm}$. (b) AFM image of isocyanate-graphene sheets on mica substrate from DMF dispersion (inset) and profile plot indicating the thickness of $\sim 1.0 \mathrm{~nm}$. (c) AFM image of graphene oxide sheets deposited on mica substrate from an aqueous dispersion and profile plot indicating the thickness of $\sim 1.0 \mathrm{~nm}$. Photographs of (d) $1 \mathrm{wt} \%$ sulfonated-graphene/TPU nanocomposite, (e) $1 \mathrm{wt} \%$ isocyanategraphene/TPU nanocomposite, (f) $1 \mathrm{wt} \%$ reduced-graphene/TPU nanocomposite, and (g) mixture of reduced-graphene in DMF. (h) Low (left) and high (right) magnification SEM cross-sectional images of sulfonated-graphene/TPU nanocomposites with 5 wt $\%$ sulfonated-graphene containing.

to these IR absorption results. First, as demonstrated by the previous works that the IR-absorption property of the graphene is mainly dependent on the integrity of its $\mathrm{sp}^{2}$ carbon network, ${ }^{28,29}$ the improved integrity of the aromatic carbon network ${ }^{28}$ of graphene materials is expected to result in stronger IR absorption for their nanocomposites. Also, truly homogeneous dispersion of graphene materials in their host polymer matrix directly correlates with their nanocomposites' effectiveness for IR absorption. TGA is an important tool for investigating the content of functional groups retaining in the graphene materials and their carbon network integrity. ${ }^{36}$ As illustrated in Figure $1 \mathrm{~d}$, the major mass loss of approximately $34 \%$ for isocyanategraphene occurs at $\sim 200{ }^{\circ} \mathrm{C}$ presumably because of pyrolysis of the labile oxygen-containing functional groups, carbamate, and amide functionalities. ${ }^{36}$ With respect to sulfonated-graphene, apart from a slight mass loss below $100{ }^{\circ} \mathrm{C}$, which can be attributed to the loss of adsorbed solvent, there is a much smaller $11 \%$ mass loss before $\sim 200{ }^{\circ} \mathrm{C}$. This suggests that the content of the functionality groups attached on sulfonated-graphene sheets is much less than that of the isocyanate-graphene and GO. ${ }^{37,38}$ Furthermore, no significant mass loss is observed for reduced-graphene even up to $400{ }^{\circ} \mathrm{C}$ implying almost complete removal of the functionality groups and enhanced integrity with improved thermal stability. These TGA results, coupled with the conductivity in the previous work ${ }^{36-38}$ and IR absorption measurements above, indeed suggest that the $\mathrm{sp}^{2}$-carbon network for the sulfonated-graphene and reduced-graphene has been restored to a greater extent than that of isocyanate-graphene.

Furthermore, as determined by AFM (Figure 2a and 2b), both sulfonated-graphene and isocyanate-graphene can be readily dispersed in DMF to form a stable dispersion composed of completely exfoliated, functionalized individual graphene sheets. This dispersion ability allows them to be intimately blended with polymer matrix through solution mixing processing leading to homogeneous dispersion throughout the polymer matrix as examined by optical images (Figure $2 \mathrm{~d}$ and $2 \mathrm{e}$ ) and scanning electron microscopy ${ }^{21}$ (SEM) (Figure 2h). Hence, the combined good dispersion and largely restored $\mathrm{sp}^{2}$ carbon network of sulfonated-graphene facilitate the remarkable absorption of IR light for its nanocompsites. However, the heavily damaged $\mathrm{sp}^{2}$ carbon network of isocyanate-graphene makes it and the corresponding nanocomposite still have poor IR absorption. 


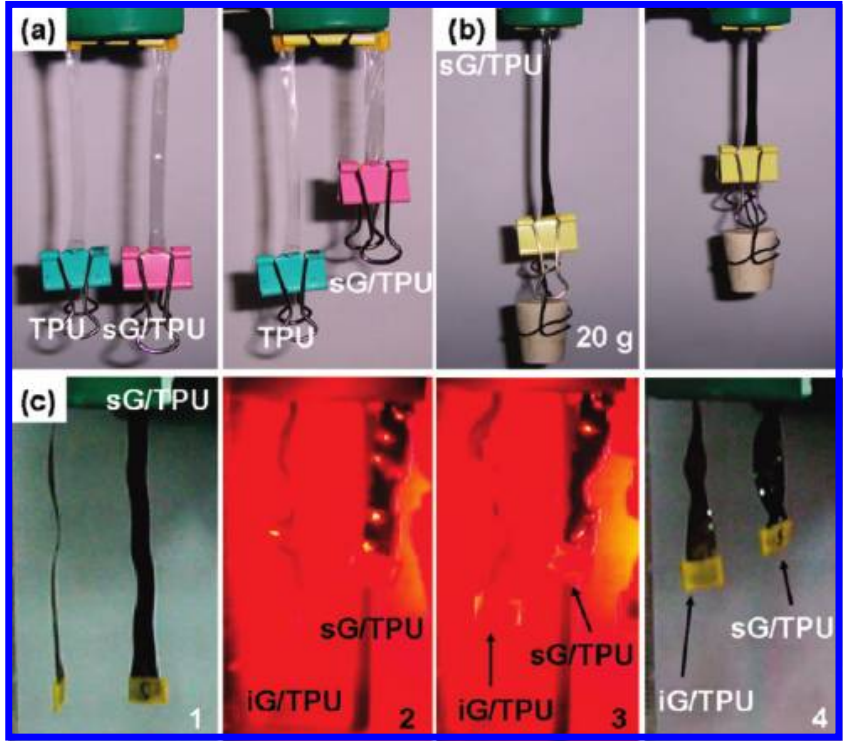

Figure 3. Optical images of IR actuation for graphene-based nanocomposites. (a) Comparison of shape recovery before (left) and after (right) remotely actuated by infrared light. Pure TPU did not recover; in contrast, $0.1 \mathrm{wt} \%$ sulfonated-graphene/TPU nanocomposites ( $\mathrm{sG} /$ TPU) $(30 \mathrm{~mm} \times 5 \mathrm{~mm} \times 0.05 \mathrm{~mm})$ contracted after actuated by infrared irradiation. (b) The $1 \mathrm{wt} \%$ sulfonated-graphene/TPU film contracted and lifted a $21.6 \mathrm{~g}$ weight $3.1 \mathrm{~cm}$ with $0.211 \mathrm{~N}$ of force on exposure to infrared light and demonstrated an estimated energy density of 0.33 $\mathrm{J} / \mathrm{g}$. (c) Comparison of IR actuation performance for isocyanategraphene/TPU (iG/TPU) and sulfonated-graphene/TPU nanocomposites with 1 wt $\%$ loading (both $30 \mathrm{~mm} \times 5 \mathrm{~mm} \times 0.05 \mathrm{~mm}$ ). (c1) The isocyanate-graphene/TPU and sulfonated-graphene/TPU films before the IR beam was turned on. (c2) The films after the IR beam was turned on for $4 \mathrm{~s}$. (c3) The films after the IR beam was turned on for $7 \mathrm{~s}$. (c4) The films after the IR beam was turned off. The sulfonated-graphene/ TPU film responded and contracted faster than the isocyanate-graphene/ TPU film toward an infrared source, and the shape recovery rate of sulfonated-graphene/TPU film was about $15 \%$ larger than that of the isocyanate-graphene/TPU film.

With respect to reduced-graphene, although it is provided with an integrated graphitic network structure, its poor solubility in the processing DMF solvent and thus rather inhomogeneous dispersion in the TPU matrix also result in relatively weak IR absorption for its nanocomposites. As displayed in Figure $2 \mathrm{f}$ and $2 \mathrm{~g}$, a mass of precipitation still can be seen even after $24 \mathrm{~h}$ sonication for reduced-graphene in DMF and, subsequently, visible coagulation can be apparently found in the reducedgraphene/TPU nanocomposite. This indicates the extremely poor dispersion of reduced-graphene in DMF as well as in the polymer matrix. Therefore, the relative perfect aromatic network of the sulfonated-graphene coupled with its homogeneous dispersion in TPU matrix results in the strongest IR absorption and, thus, best infrared-triggered actuation performance for the corresponding nanocomposite as discussed in detail below.

Figure $3 \mathrm{a}$ and $3 \mathrm{~b}$ illustrates the attractive IR-induced actuation characteristics of our sulfonated-graphene/TPU nanocomposites. The overall IR-triggered actuation process for the graphenebased nanocomposites was also recorded utilizing a video camera. The sample films were stretched to deform about $200-250 \%$ for the tests. When exposed to IR source $(\sim 30 \mathrm{~mW} /$ $\mathrm{cm}^{2}$ ), pure TPU essentially infrared transparent remains unaffected and does not contract. In contrast, sulfonated-graphene/ TPU nanocomposites (sG/TPU) with 0.1 wt $\%$ sulfonatedgraphene deformed and contracted almost completely to the original shape after actuated by IR light within $10 \mathrm{~s}$ (Figure 3a). Additionally, the sulfonated-graphene/TPU film with $1 \mathrm{wt}$
$\%$ sulfonated-graphene contracted and could lift a $21.6 \mathrm{~g}$ weight $3.1 \mathrm{~cm}$ with remarkable $0.21 \mathrm{~N}$ of force and demonstrated an estimated energy density of over $0.33 \mathrm{~J} / \mathrm{g}$ (Figure $3 \mathrm{~b}$ ). In some cycling tests, the energy density can reach as high as $0.40 \mathrm{~J} / \mathrm{g}$. Collectively, this result even outperforms many commercial elastomer actuators. ${ }^{39}$ The sulfonated-graphene, which is provided with relative perfect aromatic network and stable dispersion state in polymer matrix, can efficiently absorb and transform IR light into thermal energy and can serve as a nanoscale heater and energy-transfer unit through its homogeneous network to heat the TPU matrix uniformly and rapidly. Previous works have shown that well-dispersed graphene materials can form a percolation network in the polymer matrix at very low loadings of graphene materials. ${ }^{21}$ The absorbed thermal energy then raises the internal temperature, melting the strain-induced TPU polymer crystallites, which act as physical cross-links that secure the deformed shape, and remotely triggers the shape recovery. ${ }^{1,16}$

The IR-triggered actuation behavior of isocyanate-graphene/ TPU and reduced-graphene/TPU films was also investigated, but much poorer overall performance was observed. As presented in Figure 3c, the sulfonated-graphene/TPU responded and contracted faster than the isocyanate-graphene/TPU film, and the shape recovery rate of sulfonated-graphene/TPU film was about $15 \%$ larger than that of the isocyanate-graphene/TPU film (see Figure S1). Similar results for reduced-graphene/TPU, that sulfonated-graphene/TPU has better IR-triggered actuation performance than that of reduced-graphene/TPU, were also observed (see Figure S1). Such a phenomenon is consistent with the normalized IR absorption spectra, the structure integrity, and the dispersion ability of these three graphene materials as discussed above. Although the integrity of the aromatic network of the reduced-graphene is even better than that of the sulfonated-graphene and the isocyanate-graphene can also be well distributed in the polymer matrix, the poor dispersion for reduced-graphene and the numerous "defects" in the isocyanategraphene sheets are hampering their overall actuation performance.

Moreover, to demonstrate the repeatability of this IR-induced actuation behavior, the cycling test for the sulfonated-graphene/ TPU nanocomposites with $1 \mathrm{wt} \%$ loading was investigated as depicted in Figure 4a. The remarkable energy densities and recovery rate for these sample films were observed to sustain stabilization after 10 cycles without significant attenuation indicating repeatable response to IR light. In all cases examined, the energy densities and the recovery rate fluctuated between $0.33-0.40 \mathrm{~J} / \mathrm{g}$ and $88-95 \%$, respectively. This IR-induced actuation performance was also found to be dependent on the concentration of sulfonated-graphene as the energy densities and shape recovery rate increased with the increasing contents of sulfonated-graphene. Moreover, as can be seen in Figure 4b, both the energy densities and the shape recovery rate increased with increasing graphene loading. Since more sulfonatedgraphene nanofillers correspond to more nanoscale heater, ${ }^{16}$ higher thermal energy would be generated in the composites with higher loading, which thus could lead to better IR-induced actuation performance. ${ }^{16}$ However, this increased IR-triggered actuation performance with more loading was only observed up to a limit (1 wt \%). No obvious overall actuation performance improvement was observed with more than $1 \mathrm{wt} \%$ loadings. The reasons behind this need to be further understood.

Additionally, it has been predicted that the maximum stress generated by an actuator can be estimated as $50 \%$ of the breaking stress. ${ }^{40}$ In our cases, the sulfonated-graphene/TPU nanocomposites also show significant improved mechanical properties as presented in Figure 5. With increasing concentra- 


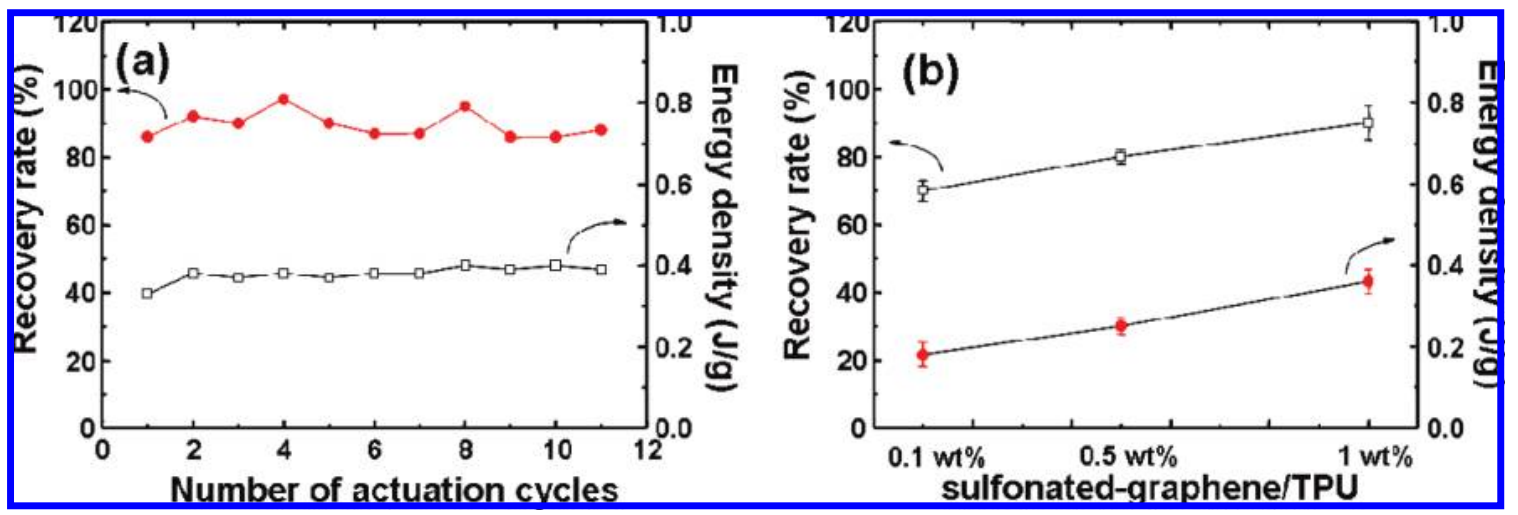

Figure 4. IR-triggered actuation performance for the sulfonated-graphene/TPU nanocomposites. (a) Recovery rate (left axis) and energy densities (right axis) for the IR-induced actuation behavior of sulfonated-graphene/TPU with $1 \mathrm{wt} \%$ loading for 11 cycles. (b) Recovery rate (left axis) and energy densities (right axis) for the IR-induced actuation behavior of sulfonated-graphene/TPU with 0.1 wt $\%, 0.5$ wt $\%$, and 1 wt $\%$ loading, respectively. The weights lifted by all the samples are about $21.6 \mathrm{~g}$.

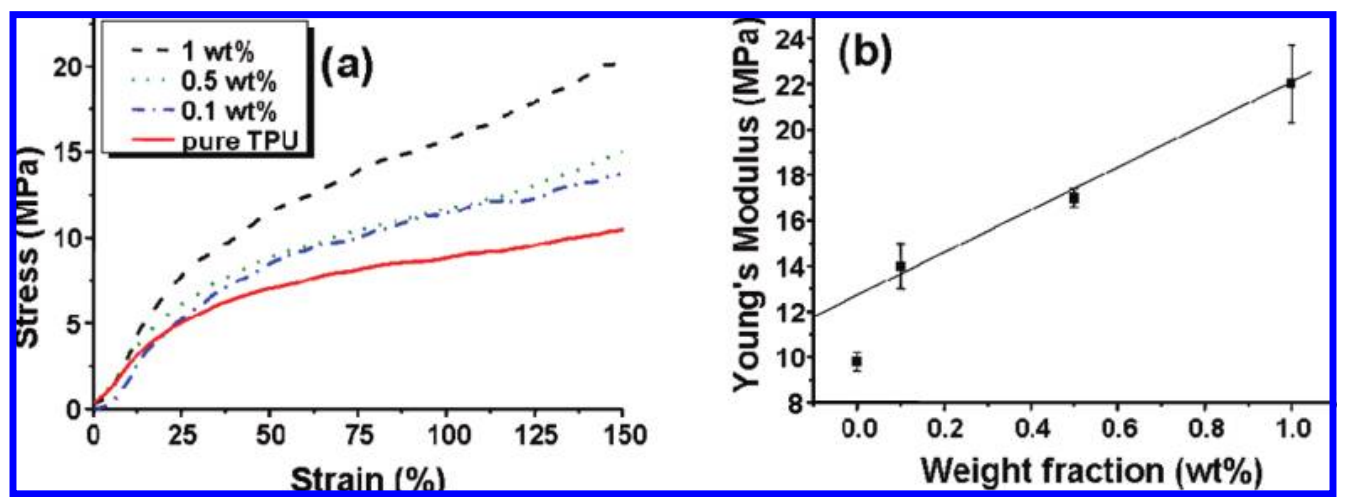

Figure 5. Mechanical performance of the sulfonated-graphene/TPU nanocomposites. (a) Representative stress-strain behavior for sulfonatedgraphene/TPU nanocomposites with different sulfonated-graphene weight loadings. (b) Young's modulus for sulfonated-graphene/TPU nanocomposites as a function of the sulfonated-graphene concentration.

tion of sulfonated-graphene, the sulfonated-graphene/TPU nanocomposites become stronger and stiffer and, significantly, the tensile stress is increased by $75 \%$ at the strain of $100 \%$ and the Young's modulus is enhanced by $120 \%$ for only $1 \mathrm{wt} \%$ loading of sulfonated-graphene. It is well-known that the agglomeration and poor dispersion of fillers in nanocomposites cause crack initiation and propagation, which would result in decrease of tensile strength for the materials. ${ }^{18}$ Thus, the observed increased tensile strength with increasing concentration of sulfonatedgraphene could be considered as indirect evidence of the homogeneous dispersion of sulfonated-graphene in the TPU matrix. Moreover, the noticeable increase of Young's modulus with such low loading of fillers, which is comparable or even rather larger than related studies, ${ }^{1,18,41}$ makes these nanocomposites highly promising in the realm of multifunctional materials.

\section{Conclusions}

In summary, utilizing graphene materials in novel ways, such as optical-absorption and nanoscale energy transfer unit, imparts additional infrared-triggered actuation behavior to the TPU matrix. The good solubility and largely restored aromatic network of sulfonated-graphene make it an excellent choice as a light-triggered actuation filler material. Graphene/TPU nanocomposites with 1 wt \% sulfonated-graphene exhibit intriguing and repeatable infrared-triggered actuation performance which can contract and lift a $21.6 \mathrm{~g}$ weight $3.1 \mathrm{~cm}$ with $0.21 \mathrm{~N}$ of force on exposure to infrared light, and the best energy density can reach as high as $0.40 \mathrm{~J} / \mathrm{g}$. Moreover, significant improvement in mechanical performance is also obtained for the graphene/ TPU nanocomposites at rather low concentrations of sulfonatedgraphene. Given these properties and the abundant supply of graphite and the processability of functionalized graphene sheets with high purity, graphene materials may open new vistas for practical applications of actuation materials.

Acknowledgment. The authors gratefully acknowledge the financial support from the NSFC (\#20774047), MOST (\#2006CB932702), MOE (\#708020) of China, and NSF of Tianjin City (\#07JCYBJC03000 and \#08JCZDJC25300).

Supporting Information Available: The preparation process of GO, isocyanate-graphene, sulfonated-graphene, and reducedgraphene. The detailed preparation method for the sample with 1 wt \% loading of sulfonated-graphene. Comparison of IR actuation performance for reduced-graphene/TPU and sulfonated-graphene/TPU nanocomposites with $1 \mathrm{wt} \%$ loading. This material is available free of charge via the Internet at http:// pubs.acs.org.

\section{References and Notes}

(1) Koerner, H.; Price, G.; Pearce, N. A.; Alexander, M.; Vaia, R. A. Nat. Mater. 2004, 3, 115.

(2) Ahir, S. V.; Terentjev, E. M. Nat. Mater. 2005, 4, 491.

(3) Lu, S. X.; Panchapakesan, B. Nanotechnology 2007, 18, 305502.

(4) Huber, J. E.; Fleck, N. A.; Ashby, M. F. Proc. R. Soc. London. Ser. A 1997, 453, 2185.

(5) Osada, Y.; Okuzaki, H.; Hori, H. Nature 1992, 355, 242.

(6) Li, F. K.; Qi, L. Y.; Yang, J. P.; Xu, M.; Luo, X. L.; Ma, D. Z. J. Appl. Polvm. Sci. 2000, 75, 68. 
(7) Tobushi, H.; Hara, H.; Yamada, E.; Hayashi, S. Smart Mater. Struct. 1996, 5,483 . 2218.

(8) Shankar, R.; Ghosh, T. K.; Spontak, R. J. Adv. Mater. 2007, 19,

(9) Jiang, H. Y.; Kelch, S.; Lendlein, A. Adv. Mater. 2006, 18, 1471.

(10) Collin, J. P.; Etrich-Buchecker, C.; Gavina, P.; Jimenez-Molero, M. C.; Sauvage, J. P. Acc. Chem. Res. 2001, 34, 477.

(11) Cho, J. W.; Kim, J. W.; Jung, Y. C.; Goo, N. S. Macromol. Rapid Commun. 2005, 26, 412.

(12) Pelrine, R.; Kornbluh, R.; Pei, Q. B.; Joseph, J. Science 2000, 287, 836

(13) Cviklinski, J.; Tajbakhsh, A. R.; Terentjev, E. M. Eur. Phvs. J. E 2002, 9, 427.

(14) Lendlein, A.; Jiang, H. Y.; Junger, O.; Langer, R. Nature 2005, 434,879 .

(15) Ahir, S. V.; Squires, A. M.; Tajbakhsh, A. R.; Terentjev, E. M. Phvs. Rev. B 2006, 73, 085420

(16) Yang, L. Q.; Setyowati, K.; Li, A.; Gong, S. Q.; Chen, J. Adv. Mater. 2008, 20, 2271.

(17) Lendlein, A.; Kelch, S. Angew. Chem., Int. Ed. 2002, 41, 2034.

(18) Auad, M. L.; Contos, V. S.; Nutt, S.; Aranguren, M. I.; Marcovich, N. E. Polvm. Int. 2008, 57, 651

(19) Novoselov, K. S.; Geim, A. K.; Morozov, S. V.; Jiang, D.; Zhang,

Y.; Dubonos, S. V.; Grigorieva, I. V.; Firsov, A. A. Science 2004, 36, 666.

(20) Avouris, P.; Chen, Z. H.; Perebeinos, V. Nat. Nanotechnol. 2007, 2,605 .

(21) Stankovich, S.; Dikin, D. A.; Dommett, G. H. B.; Kohlhaas, K. M.; Zimney, E. J.; Stach, E. A.; Piner, R. D.; Nguyen, S. T.; Ruoff, R. S. Nature 2006, 442, 282

(22) Gomez-Navarro, C.; Weitz, R. T.; Bittner, A. M.; Scolari, M.; Mews, A.; Burghard, M.; Kern, K. Nano Lett. 2007, 7, 3499

(23) McAllister, M. J.; Li, J. L.; Adamson, D. H.; Schniepp, H. C.; Abdala, A. A.; Liu, J.; Herrera-Alonso, M.; Milius, D. L.; Car, R.; Prud'homme, R. K.; Aksay, I. A. Chem. Mater. 2007, 19, 4396.

(24) Ramanathan, T.; Abdala, A. A.; Stankovich, S.; Dikin, D. A.; Herrera-Alonso, M.; Piner, R. D.; Adamson, D. H.; Schniepp, H. C.; Chen, X.; Ruoff, R. S.; Nguyen, S. T.; Aksay, I. A.; Prud'homme, R. K.; Brinson, L. C. Nat. Nanotechnol. 2008, 3, 327.
(25) Gomez-Navarro, C.; Burghard, M.; Kern, K. Nano Lett. 2008, 8, 2045

(26) Booth, T. J.; Blake, P.; Nair, R. R.; Jiang, D.; Hill, E. W.; Bangert, U.; Bleloch, A.; Gass, M.; Novoselov, K. S.; Katsnelson, M. I.; Geim, A. K. Nano Lett. 2008, 8, 2442.

(27) Lee, C. G.; Wei, X. D.; Kysar, J. W.; Hone, J. Science 2007, 321, 385 .

(28) Sun, X. M.; Liu, Z.; Welsher, K.; Robinson, J. T.; Goodwin, A.; Zaric, S.; Dai, H. J. Nano Res. 2008, 1, 203.

(29) Becerril, H. A.; Mao, J.; Liu, Z.; Stoltenberg, R. M.; Bao, Z.; Chen, Y. ACS Nano 2008, 2, 463.

(30) Balandin, A. A.; Ghosh, S.; Bao, W. Z.; Calizo, I.; Teweldebrhan, D.; Miao, F.; Lau, C. N. Nano Lett. 2008, 8, 902.

(31) Liu, Z. F.; Liu, Q.; Huang, Y.; Ma, Y. F.; Yin, S. G.; Zhang, X. Y.; Sun, W.; Chen, Y. S. Adv. Mater. 2008, 20, 3924.

(32) Li, D.; Muller, M. B.; Gilje, S.; Kaner, R. B.; Wallace, G. G. Nat. Nanotechnol. 2008, 3, 101.

(33) Niyogi, S.; Bekyarova, E.; Itkis, M. E.; McWilliams, J. L.; Hamon, M. A.; Haddon, R. C. J. Am. Chem. Soc. 2006, 128, 7720

(34) Stankovich, S.; Piner, R. D.; Chen, X. Q.; Wu, N. Q.; Nguyen, S. T.; Ruoff, R. S. J. Mater. Chem. 2006, 16, 155.

(35) Schniepp, H. C.; Li, J. L.; McAllister, M. J.; Sai, H.; HerreraAlonso, M.; Adamson, D. H.; Prud'homme, R. K.; Car, R.; Saville, D. A.; Aksay, I. A. J. Phys. Chem. B 2006, 110, 8535.

(36) Stankovich, S.; Piner, R. D.; Nguyen, S. T.; Ruoff, R. S. Carbon 2006, 44, 3342 .

(37) Si, Y. C.; Samulski, E. T. Nano Lett. 2008, 8, 1679.

(38) Stankovich, S.; Dikin, D. A.; Piner, R. D.; Kohlhaas, K. A.; Kleinhammes, A.; Jia, Y.; Wu, Y.; Nguyen, S. T.; Ruoff, R. S. Carbon 2007, 45, 1558.

(39) Pelrine, R.; Kornbluh, R.; Joseph, J.; Heydt, R.; Pei, Q. B.; Chiba, S. Mater. Sci. Eng. C: Biom. Supramol. Sust. 2000, 11, 89.

(40) Spinks, G. M.; Mottaghitalab, V.; Bahrami-Saniani, M.; Whitten, P. G.; Wallace, G. G. Adv. Mater. 2006, 18, 637.

(41) Gall, K.; Dunn, M. L.; Liu, Y. P.; Finch, M.; Lake, N. A. Acta Mater. 2002, 50, 5115.

JP901284D 